\title{
Anatomy and physiology of anorectum: the hypothesis of fecal retention, and defecation
}

\author{
(1) MICHAEL LEVIN 1,2 \\ ${ }^{1}$ Department of Pediatric Radiology, 1st State Hospital, Minsk, Belarus \\ ${ }^{2}$ Dorot-Netanya Geriatric Medical Center, Israel
}

\section{ABSTRACT}

Continence and defecation are two essential functions of the human body. The normal anatomy of the anorectum is well documented in the literature. The data on the physiology of these processes are controversial and sketchy. The article discusses scientific facts that describe the different aspects of the physiology of anorectum. On this basis, the hypothesis of fecal retention and defecation in the form of successive reflex reactions of the rectum, and pelvic floor muscles, including the internal anal sphincter, external anal sphincter, puborectalis muscle, and levator plates, was proposed. This hypothesis allows for explaining the pathophysiology of functional constipation, fecal incontinence, anorectal malformations, etc.

Keywords: Anal canal length; anal sphincters; defecation; fecal retention; hypothesis physiology anorectum.

\section{INTRODUCTION}

Continence and defecation are two essential functions of the human body. Continence is the ability to retain feces until an acceptable time for defecation. Defecation is the evacuation of fecal material from the colon. Both functions involve complex physiologic processes that are not completely understood. ${ }^{1}$ The normal anatomy of the gastrointestinal tract is well documented in the literature. The data on the physiology of these processes are controversial and sketchy. For example, muscular contraction is accompanied by energy expenditure and resource depletion. All the known muscles, including the heart, relax after the contraction. During the relaxation, their contractile capacity is recovered. It is still not clear how the anal canal is in the continuous contraction, and which muscles are involved in the act of defecation. It is known that the external anal sphincter (EAS) is responsible for the emergency continence during the increase of abdominal pressure. However, the intensity of contraction promptly decreases after 8-15 seconds. ${ }^{2,3}$ It is hard to ascribe the continence to continuous contraction of the internal anal sphincter (IAS).

A clear understanding of the normal physiology of the anorectal zone could significantly improve our approach to such pathological conditions like chronic constipation, fecal incontinence, anorectal malformations, etc.

This work presents a new hypothesis of the continence and defecation, based on analysis of the literature and our own studies.

\section{MATERIALS FOR ANALYSIS}

Colon; the continence starts above of the rectum. Only the distal part of the sigmoid colon was available for functional manometric examination. Between the descending and sigmoid colon there 
is a physiological "colosigmoid sphincter" (CSS) a length of $2.1 \pm 0.9 \mathrm{~cm}$. In this zone, the pressure was significantly higher than in the adjacent segments. After a quick inflation of the balloon of large diameter in the descending colon, the decrease of pressure in the CSS was noted. After inflating the same balloon in the sigmoid colon, the pressure in the CSS increases. On the other hand, inflation with a small balloon did not affects the tone of this sphincter. The CSS is involved in a passage of large intestinal contents. CSS retains the feces until it reaches a certain volume. ${ }^{4}$ Between the rectum and sigma is located the intestinal segment of $2.8 \pm 0.9 \mathrm{~cm}$ length with the layer of circular muscle thickened in comparison to the segments above and below it. ${ }^{5}$ While the pressure in the sigmoid colon increases, this functional rectosigmoid sphincter (RSS) relaxes. In response to increased pressure in the rectum, its tonus rises. ${ }^{6}$ Different reactions of RSS to different volume of the balloon were found. After a quick inflation of the balloon in the sigma with $52.1 \pm 3.6 \mathrm{ml}$ of liquid, the tone of the RSS increased but the rectal pressure did not change. After the rapid inflation into the balloon of $86 \pm 4.1 \mathrm{ml}$, the RSS relaxed, and the balloon was dispelled to the rectum. It was accompanied by an increase of the rectal pressure, and the balloon was expelled. ${ }^{7}$ Thus, the RSS with CSS prevent the rapid advance of the large volume of the feces from the colon into the rectum. They are the first level of the continence.

Rectum is a reservoir where more dense portions of feces are collected than in the colon, as well as it is involved in the process of defecation by strong contractions. ${ }^{1}$ It is in the pelvis retroperitoneally. It starts from the third sacral vertebrae and ends at the level of the pubococcygeal (P-C) line, where it borders on the anal canal. From a functional point of view, the rectum begins caudally to the RSS. While fecal retention, it performs a cumulative function (Figure 1), and during bowel movements, its strong peristaltic wave, which starts from the RSS, expels the stool through the open anal canal. Table 1 shows the normal width of the rectum and the anal canal in patients of different ages after filling the colon with barium at least to the splenic angle. ${ }^{8,9}$

At least starting in the descending colon, small soft fecal boluses are delivered in batches above the CSS, where they coalesce into a single bolus that gradually decreases in volume as fluid is absorbed by the intestinal wall. However, as more portions arrive, the total stool volume reaches a certain level, which causes a rise in pressure. It causes the CSS to open and the peristaltic wave pushes the denser bolus into the sigmoid colon. The same process occurs in the sigmoid and rectum, because of which lumpy dense feces accumulate over the anal canal. Obviously, the same process occurs along the entire length of the colon. In children, the slow movement of the bolus occurs without the participation of sphincters. In adults, in addition to CSS and RSS, 8 more functional sphincters are described. They are not always simultaneous (Figure 2). ${ }^{10}$

Colon sphincters arise during ontogenesis. They manifest as a limited thickening of the circular muscle fibers. As a rule, 2-3 haustras in these places are underdeveloped. At present, it is difficult to determine whether functional sphincters are present in healthy individuals or only in intestinal pathology. It is likely that dyskinesia of these sphincters plays an important role in the development of chronic constipation and diverticula of the left half of the colon. ${ }^{11}$

With or without sphincters the slow peristalsis of the colon, during which fluid is absorbed from the feces, plays a significant
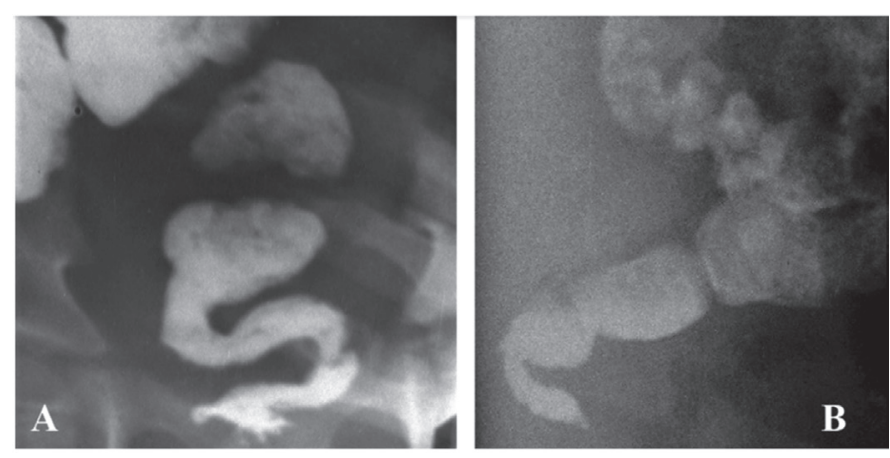

Figure 1. The radiographs of the anorectum of an 8-year-old child performed 24 hours after oral administration of barium (passage). (A). Frontal radiograph. (B). Lateral radiograph. During the slow promotion across the rectum, the fecal portions decrease in volume and become more contrast (denser) as a result of fluid absorption by the intestinal wall. Most of the feces are in the sigmoid colon.

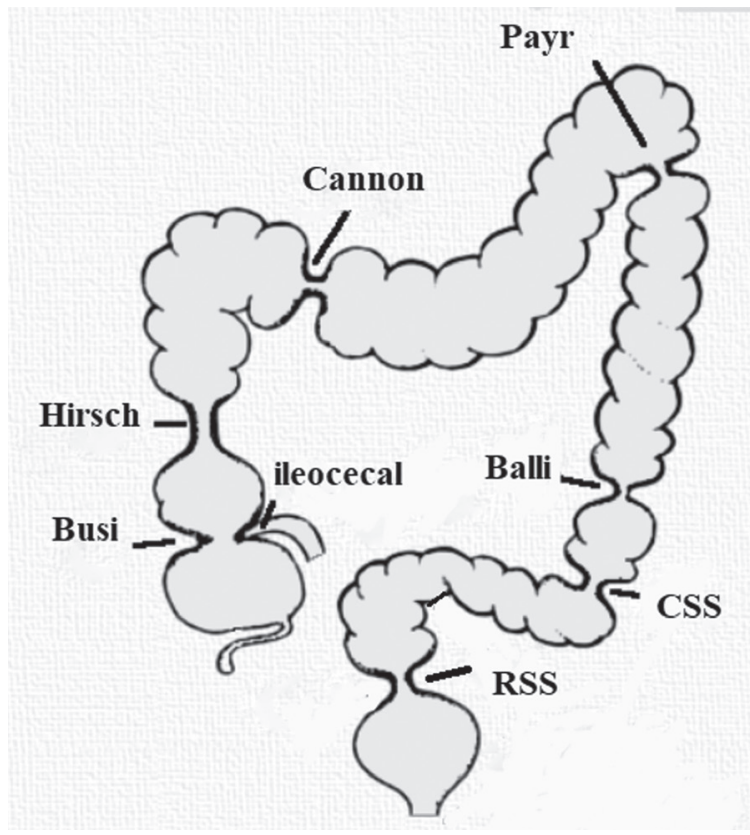

Figure 2. Functional sphincters of the colon RSS: Rectosigmoid sphincter; CSS: Colosigmoid sphincter 
role in long-term fecal retention. The anal canal starts from the $\mathrm{P}$-C line and continues to the anus. Its length is given in Table 1. The anal canal consists of several elements, each of which plays an important role in fecal retention and defecation. In its center is the smooth muscle of the IAS, which is a continuation of the circular layer of the rectum. However, IAS are both morphologically and functionally different from the circular layer of the rectum. Alamovich et al. investigated the normal innervation of the IAS. This study shows that the IAS itself has no autonomous innervation unlike the rest of the digestive tube. ${ }^{12}$ During a bowel movement, it opens all along the length, completely and simultaneously. For 24 hours a day, except for a few seconds, during a bowel movement, the anal canal is in a closed position, preventing leaking of the stool and gas. High pressure in the anal canal $\left(25-85 \mathrm{~cm} \mathrm{H}_{2} \mathrm{O}\right)$ compared with the pressure in the rectum $\left(2-5 \mathrm{~cm} \mathrm{H}_{2} \mathrm{O}\right)$ is the result of contraction of the IAS, puborectalis muscle (PRM), and EAS. ${ }^{13}$ It is considered that at rest, the IAS is in a state of partial tetanus and contributes approximately $55 \%$ of the anal resting pressure. ${ }^{14}$

\begin{tabular}{|c|c|c|}
\hline Ages & $\begin{array}{l}\text { The width of the } \\
\text { rectum }(\mathrm{cm})\end{array}$ & $\begin{array}{l}\text { The length of the } \\
\text { anal canal }(\mathrm{cm})\end{array}$ \\
\hline 5 days -11 months & $1.3-3.0(2.24 \pm 0.09)$ & $1.7-2.5(2.21 \pm 0.15)$ \\
\hline $1-3$ years & $3.0-3.7(3.21 \pm 0.11)$ & $2.3-2.8(2.55 \pm 0.10)$ \\
\hline $4-7$ years & $3.0-3.9(3.43 \pm 0.14)$ & $2.5-3.6(3.17 \pm 0.14)$ \\
\hline $8-10$ years & $3.2-4.1(3.72 \pm 0.05)$ & $2.6-3.7(3.11 \pm 0.10)$ \\
\hline $11-15$ years & $3.6-4.6(3.95 \pm 0.07)$ & $3.1-3.9(3.43 \pm 0.10)$ \\
\hline $23-64$ years & $3.5-4.8(3.95 \pm 0.21)$ & $3.4-4.2(4.08 \pm 0.07)$ \\
\hline
\end{tabular}

Longitudinal fibers between the IAS and EAS consists primarily of smooth muscle fibers that continued from the longitudinal muscle of the rectum. The levator ani muscle (LAM) attached directly to the lateral surface of the longitudinal smooth muscle of the rectum. In the lateral and posterior position, the LAM partially overlapped the EAS. ${ }^{15}$

The IAS is in the center of the pelvic floor muscles, which fan out from it and are attached to the pelvic ring. Muscle complex which forms a pelvic diaphragm is subdivided into four muscles: pubococcygeus, iliococcygeus, coccygeus, and puborectalis, which are located on both sides of the median line. The first three of these four muscles, often called LAM or levator plates, are attached peripherally to the pubic bones, the ischial bones, and to the arcus tendinous, as a thickened part of the obturator fascia. The inner ends of muscle fibers of the LAM are intimately connected with longitudinal muscle and with the deep portion of the EAS on the side and posterior walls of the anal canal. ${ }^{13,16,17}$ The PRM is a strong, U-shaped loop of striated muscle that sling the anorectal junction to the posterior aspect of the pubis. Tonic contraction of PRM leads to the appearance of an acute anorectal angle. ${ }^{16-18}$ Muscles, in general, have a relatively simple function; they shorten as they contract. Since LAM and PRM have different muscle attachments, they perform different functions. During PRM contraction, the cranial part of the anal canal is pulled forward, resulting in compression of the posterior wall of the anal canal and an acute anorectal angle will occur. Raizada et al. ${ }^{19}$ showed that with voluntary contraction, pressures increase significantly in the cranial part of the anal canal that is surrounded by the PRM. They concluded that it is an important mechanism by which PRM contributes to the anal continence mechanism. "Most investigators agree that the PRM is a key component of anal continence mechanism" ${ }^{19}$

Since the posterior ends of the LAM are attached along the posterior semicircle of the pelvic ring, and the anterior ends are attached to the posterior and lateral walls of the longitudinal muscle and EAS, then during LAM contraction, the posterior and lateral walls of the anal canal are stretched, revealing the anal canal to the width of the rectum, which is accompanied by the disappearance of the anorectal angle. ${ }^{20,21}$ Shafik and El-Sibai ${ }^{22}$ discovered that the electrical stimulation of the LAM and the PRM by the needle electrode does not change the pressure in the empty rectum. However, when the balloon of $156.6 \pm 34.3 \mathrm{ml}$ is inserted - average pressure in the rectum increased to $64.6 \pm 18.7$ $\mathrm{cm} \mathrm{H}_{2} \mathrm{O}$, the need to defecate appeared, and the balloon was expelled. ${ }^{22}$ Since electrical stimulation causes muscle contraction, this suggests that defecation accompanied by a contraction, rather than relaxing of the LAM. Li and $\mathrm{GuO}^{23}$ showed by the use of CT defecography, that the LAM's main function is to open the genital hiatus and the anus during defecation. Bush et al. ${ }^{24}$ studied normal defecation with video proctography or magnetic resonance imaging. They showed that three muscle vectors open the anorectal angle prior to defecation, causing the anorectal luminal diameter to increase to approximately twice its resting size. These vectors are forwards (anterior wall), backward, and downwards (posterior wall). If the effective diameter of the anus is doubled during defecation, the frictional resistance is reduced by a factor of 8 . These studies suggest that the opening of the anal canal during defecation is an active process because of the contraction of LAM. However, there is still no consensus regarding the understanding of normal defecation and the role of LAM. ${ }^{19,25,26}$

The EAS is the elliptical cylinder of striated muscle that envelops the entire length of the inner tube of smooth muscle, but it ends 
slightly more distal than the IAS. It consists of three parts: deep, superficial, and subcutaneous. The deepest part of the EAS is intimately related to the puborectalis muscle. The anatomy of the anorectal region is presented in the diagram (Figure 3).

In the anal canal activity is the intermittent, transient relaxation of the IAS, which allows to descent of distal rectal contents into the upper anal canal, endowing a perception of their physical nature. This so-called sampling reflex occurs approximately seven times per hour. This reflex can be reproduced under laboratory condition, where rectal distention cause reflex relaxation of the IAS, as well as contraction of the EAS and PRM (anorectal inhibitory reflex). ${ }^{25}$

In an X-ray examination, the anal canal is defined as the contraction zone between barium in the rectum and the radiopaque marker, which is located next to the anus (Figure 4A). Levin and $\operatorname{Troyan}^{20}$. measured the pressure in the anal canal during a barium enema. The penetration of barium into the anal canal in front of the tip of the enema was observed from one to three times. During the appearance of barium in the anal canal, the anal pressure decreased, and after the disappearance of barium, it returned to the basal level. These observations allowed you to draw two conclusions: (1) the expansion of the rectum causes reflex relaxation of IAS with a simultaneous contraction of EAS and PRM; (2) the penetration of barium into the upper part of the anal canal in front of the tip of the enema is the radiological equivalent of the anorectal inhibitory reflex.

The healthy rectum is compliant, i.e. it can accommodate significant volume of stool with little changes in pressure. ${ }^{25}$ Musial and Crowell ${ }^{26}$ showed an adaptive response of the rectum to distention and its role in the determination of rectal sensory perception thresholds. ${ }^{24}$ The rectum, adapting to an

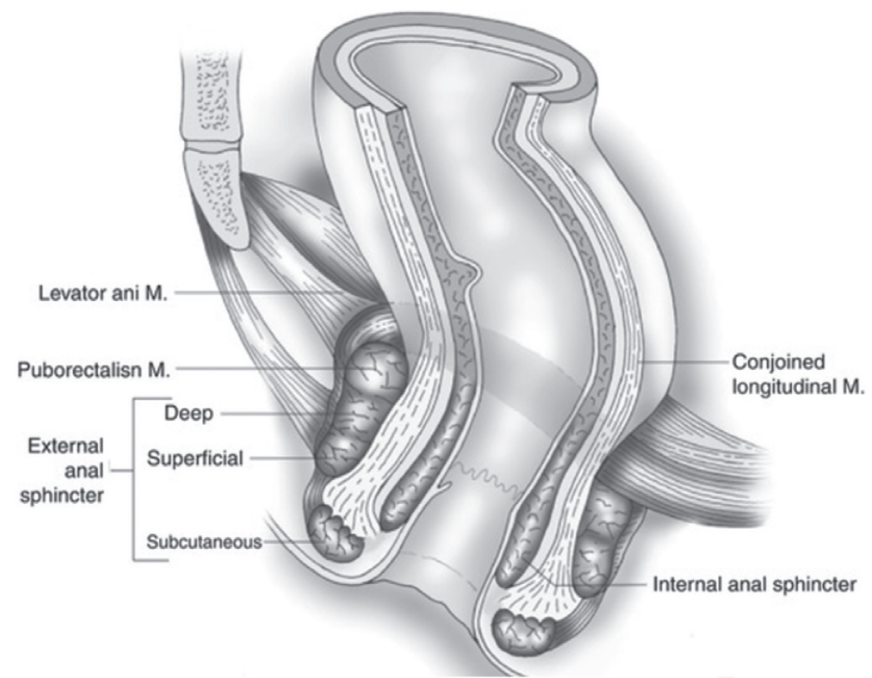

Figure 3. Muscles of the anal canal from an article by Jorge and Habr - Gama ${ }^{18}$ ever-increasing volume of contents, relaxes, because of which the pressure in it does not increase. However, adaptability has limitations both in volume and in rectal pressure. With a certain amount of rectal contents, a need for defecation arises, and defecation itself arises when the abdominal wall and the diaphragm are strained, which leads to additional an increase in rectal pressure. Rectal pressure depends on the volume of the feces, and on the tone rectal wall. Sensitive elements in the wall of the rectum are affected by two forces directed towards each other: wall extension with fecal masses and the tone of the rectal wall. The greater the tone of the rectum, the smaller need for the volume of feces, at which a desire for a bowel movement and the possibility of its implementation arises. For example, the high tone of the rectum with diarrhea causes the need for defecation with scanty amounts of feces. The last example proves that sensitive elements in the wall of the rectum do not respond to stretching and changes of their spatial location, but to the rectal pressure. The networks of pacemaker (interstitial cells of (ajal) coordinates the motor function of the rectum. ${ }^{27}$

Contraction of the muscle fibers is a "work" accompanied by energy consumption, without its renewal muscle cannot continue to contract. Following the contraction inevitably the muscle relaxation must arise, during which the muscle restores its capacity for subsequent contraction. The ability of the IAS to prolonged and continuous contraction can be explained by the

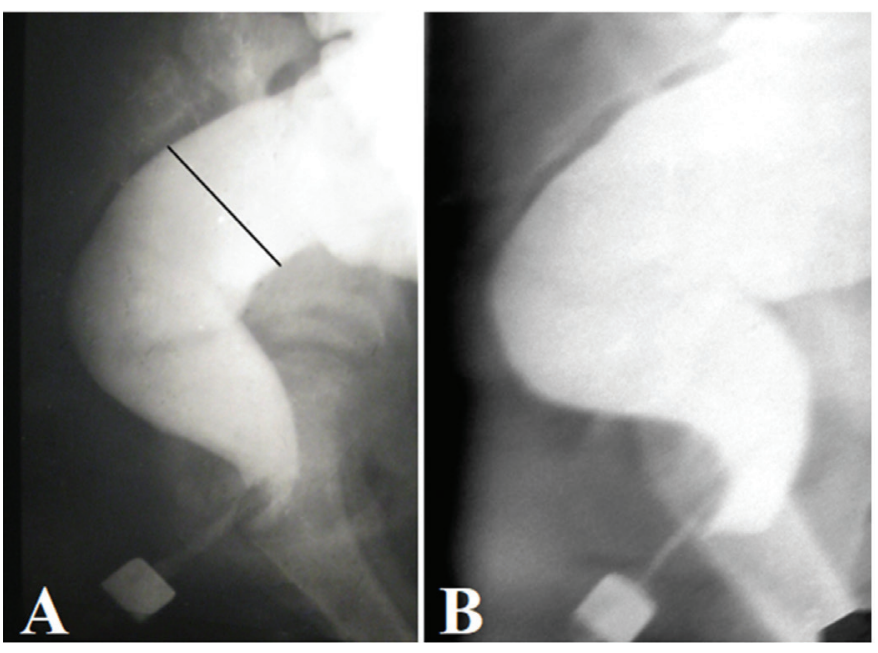

Figure 4. Lateral radiographs of anorectum during a barium enema. (A). The true length of the anal canal is equal to the distance between the rectum and the marker, multiplied by a distortion coefficient equal to the ratio of the true diameter of the marker $(1.6 \mathrm{~cm})$ to the width of its image on the radiograph. (B). During the study, barium penetrates periodically into the anal canal in front of the tip of the enema. At this time, the posterior wall of the anal canal is always pressed against the tip by the contracted PRM. After a few seconds, barium disappears from the anal canal. It is squeezed into the rectum because of a contraction of IAS

PRM: Puborectalis muscle; IAS: Internal anal sphincter 
fact that the muscle bundles do not contract simultaneously. It is known that the sheaves of smooth muscle fibers anastomosing with each other forming a tightly knit group of fibers, which operate together. ${ }^{28}$ Likely that the different groups of smooth muscle fibers in the IAS are at different stages of recovery of the contractile capacity. At any moment an electrical stimulus that excited from the cells Cajal, leads to a contraction of the groups that are ready to contract. By the next wave of electricity, other groups that have fully restored their capacity are stimulated. This continuous process provides a permanent contraction of the IAS. When there is a need to strengthen the fecal retention, extraintestinal nerve centers generate a stronger electric potential. This leads to the contraction not only of the muscle fibers group, which is ready to contract but also, the additional groups that are close to this state. The contraction of the larger number of muscle groups leads to increased pressure in the anal canal.

Skeletal muscles are capable of two types of contraction: tonic and mechanical. Tonic prolonged contraction of the LAM, PRM, and EAS explained by postural reflex..$^{29,30}$ Each nervous axon has a connection to the muscle fibers scattered throughout the muscle. Therefore, even a small amount of the contracted muscle fibers results in a contraction of the whole muscle. The muscle tone is dependent on the number of fibers participating in the contraction, i.e. from the percentage of axons activating muscle contraction. The prolonged tonic contraction is due to the continuous replacement of the axons activating the different groups of muscle fibers. At a different time, the different groups of the muscle fibers are contracted. At this moment, other muscle fibers restore their contractile potential.
The simultaneous contraction of all muscle fibers leads to a shortening of the muscle and it causes a mechanical movement of the not fixed point of attachment to the fixed point to the bone, such as, for example, the LAM contraction during defecation (Figure 5) and the PRM contraction during anorectal inhibitory reflex. During a mechanical contraction of circular EAS, anal pressure rises sharply. However, the mechanical contraction of striated muscles lasts less than one minute. ${ }^{31}$

There are no voids in the tissues of the pelvic floor. Obviously, during defecation, the anal canal occupies a large volume, not as a result of the relaxation of the sphincters. Only muscle contraction could create this wide channel. And only LAM plates could do this. Thus, the tonic contraction of the LAM provides mechanical support to the rectum and other pelvic floor viscera. ${ }^{19}$ It contracts during bowel movements, disclosing the anal canal for passing the stool. The tonic contraction of PRM and EAS together with IAS close the anal canal. Each of these sphincters contributes to the creation of basal anal pressure. Surprisingly, almost all the listed scientific data were published in a book edited by M.M. Henry and M. Swash in $1985 .{ }^{32}$

Using the above scientific data, Levin proposed the hypothesis of the motor function of the anorectum and pelvic floor. ${ }^{21}$

\section{RESULTS}

\section{The hypothesis of fecal retention and defecation}

Fecal retention: At rest, the IAS and striated muscles of the pelvis floor are in a state of tonic contraction. They help to support the pelvic organs and participate in the continuous
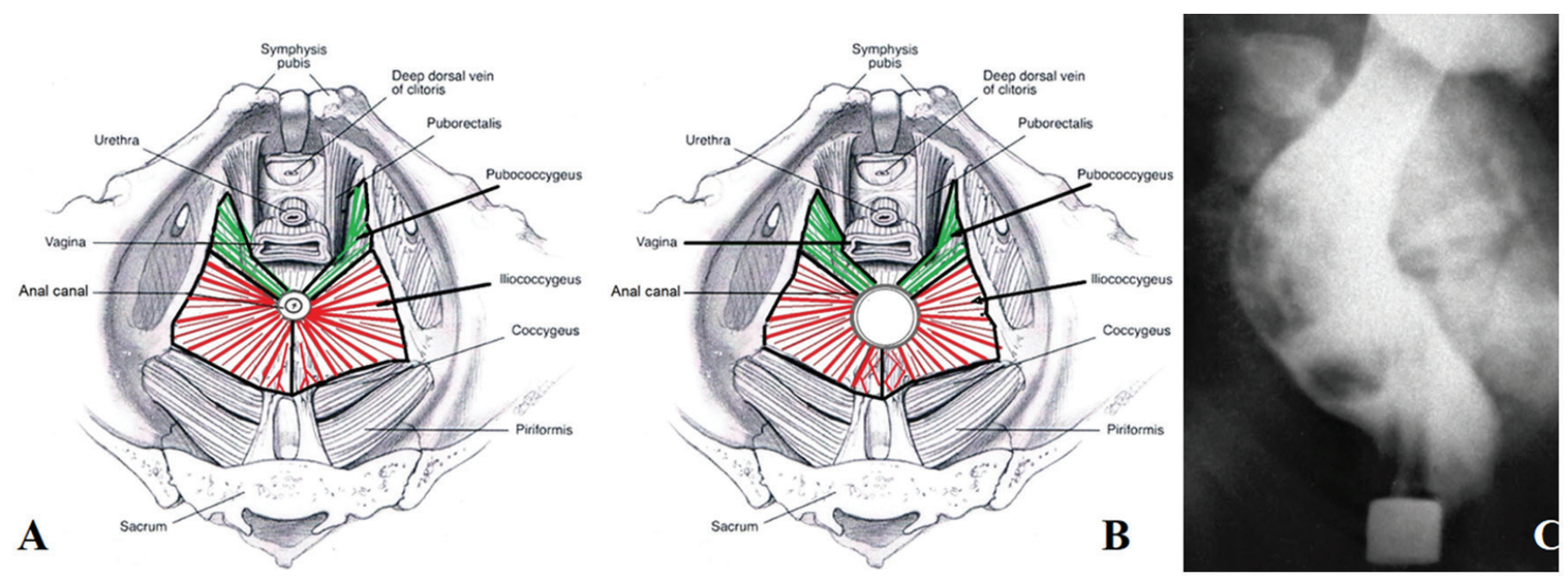

Figure 5. Scheme cross-section of the anorectal region at the level of the deep portion of the EAS. A scheme from the article of Bharucha17 was used. (A). At rest anal canal is in a closed state. (B). During defecation, the anal canal opened because of the contraction of LAM (pubococcygeus and iliococcygeus). (C) Lateral radiography of anorectum of the child during a barium enema. The moment of defecation attempt was recorded. A wide opening of the anal canal is determined. A contrast marker strung on the tip of the enema is located near the anus.

EAS: External anal sphincter; LAM: Levator ani muscle 
retention of feces. The pressure in an empty rectum is equal to the intra-abdominal pressure. It is basal rectal pressure (BRP). Its absolute indices are irrelevant, because, firstly, in different articles, they differ significantly from each other, and, secondly, intrarectal catheters distort the true functional characteristics. When the fecal bolus penetrates from the sigmoid colon into the rectum, it stretches the wall of the rectum and rectal pressure increases above the BRP. This pressure we called the threshold pressure of the first order (TP-1). It causes a reflex relaxation of the IAS and contraction of the EAS and PRM. The PRM during contraction pulls forward the upper part of the anal canal. In front, inside the PRM loop the anal pressure decrease because of the IAS relaxation. Between the rectum and anal canal, there is a narrow opening through which the gas and liquid feces can penetrate the upper part of the anal canal. In the mucosa at this level, there are sensors that allow distinguishing the liquid from the gas. The presence of gas is enough to strain the abdominal wall to increase rectal pressure and expel gas through the lower part of the closed anal canal. When the liquid feces penetrate the upper part of the anal canal the tone of the EAS increase, which leads to a contraction of the IAS and crowding out of the fluid from the anal canal into the rectum. During anorectal inhibitory reflex, the formed stool remains in the rectum due to the acute anorectal angle and the narrow hole between the rectum and anal canal. After a few seconds, the rectum adapts to the new rectal volume and relaxes. The rectal pressure drops up to BRP, resulting in the contraction of the IAS and relaxation of the PRM and EAS. After entering the rectum of another bolus of feces this picture (anorectal inhibitory reflex) is repeated. This picture can be observed up to seven per hour. During IAS relaxation, its muscle fibers restore contraction ability. In this period, the fecal retention is performed by the PRM and EAS contraction. During the rise of the intra-abdominal pressure (rise from the spot, cough, etc.), the reflex contraction of all sphincters occurs. The tone of the IAS increases since the electric potential from the center outside the rectum increases the number of contracted muscle bundles.

When the volume of stool in the rectum reaches a certain value, the rectal pressure rises from TP-1 to the threshold pressure of the second-order (TP-2), in which a need for a bowel movement appears. If this need does not coincide with the possibility of its implementation, the rectum continues to relax to a limited extent. At the same time, there is an increase in the tone of the recto-sigmoid sphincter, which prevents the further penetration of feces from the sigmoid colon into the rectum. ${ }^{33}$

Defecation: When the need for a bowel movement coincides with the possibility of its implementation, a straining of the abdominal wall and diaphragm increases, which leads to an increase in the abdominal and rectal pressure from TP- 2 to the threshold pressure of the third-order (TP-3). At the rectal pressure TP-3, the reflex defecation takes place: a strong peristaltic wave of the rectum expels stool through the open anal canal. The wide opening of the anal canal is due to the relaxation of the IAS, PRM, and EAS, with a simultaneous contraction of the LAM. Any of the pressure levels depend not only on the volume of feces but also on the tone of the rectum. During the anal canal opening, its wall is stretched at the level of deep and superficial portions of the EAS. Since the subcutaneous portion of the EAS is not connected with LAM it relaxed, but does not stretch (Figure 3). Therefore, during the evacuation of soft feces, it forms a tape, the diameter of which depends on the viscosity of the feces.

\section{Applied physiology}

1. Prerequisites for normal bowel movements: a) Normal innervation of all elements of the anal canal and preservation of reflexes. b) The maximum width of the rectum, which forms feces, should not exceed the maximum possible width of the anal canal. c) Normal LAM function is necessary for the wide disclosure of the anal canal, which provides minimal resistance to the movement of feces.

2. This hypothesis allows us to explain the "paradoxical" contraction in PRM during an attempt to defecate in constipated patients. To create pressure that provokes defecation in the wide rectum (megarectum) with a low tone of the rectal wall, the rectal balloon of significantly larger diameter is needed than in the control. The volumes of the rectal balloon that in healthy people create TP-3 pressure and provoke defecation, in patients with obstructive constipation, create TP-1 pressure, causing a contraction in PRM and EAS.

3. Therefore, the key to understanding the pathogenesis of functional constipation is in the expansion of the rectum. Delayed bowel movement leads to an increase in the diameter of the stool. An attempt to defecate causes pain and stops the following attempts. There is a vicious circle, because of which after a while there is a stable expansion of the rectum megarectum. The rectum forms fecal masses of large diameter difficult to pass through the anal canal. Wide stool stretches the muscles of the pelvic floor, causing stretching and weakening of LAM and PRM, which leads to increased constipation (weakness of LAM) and fecal incontinence (weakness of PRM). This is how descending perineum syndrome develops.

4. Measuring the width of the rectum is the key to determining the cause of constipation and its prognosis. ${ }^{8,9,11,34-36}$ 


\section{NOTES}

The normal anatomy of the gastrointestinal tract is well documented in the literature. The data on the physiology of these processes are controversial and sketchy. Knowledge of the physiology of anorectum allows us to understand the development of pathological processes in this area and apply pathophysiological treatment of chronic constipation, fecal incontinence, etc. The hypothesis presented does not contradict the known scientific facts and can serve as a basis for further research.

\section{Ethics}

Ethics Committee Approval: Since this study does not include studies in humans or animals, ethics committee approval was not obtained.

Informed Consent: Since this study does not include studies in humans or animals, informed consent was not obtained.

Peer-review: Externally peer-reviewed.

\section{DISCLOSURES}

Financial Disclosure: The author declared that this study has received no financial support.

\section{REFERENCES}

1. McCrea GL, Miaskowski C, Stotts NA, Macera L, Varma MG. Pathophysiology of constipation in the older adult. World J Gastroenterol 2008; 14: 2631-2638.

2. Meunier P, Mollard P, Jaubert de Beaujeu M. Manometric studies of anorectal disorders in infancy and childhood: an investigation of the physiopathology of continence and defaecation. Br J Surg 1976; 63: 402-407.

3. Levin MD. Rentgenofunktsional'nye issledovaniia aktov uderzhaniia i defekatsii [Roentgenofunctional studies of feces retention and defecation]. Pediatriia 1983; 2: 49-52.

4. Shafik A, Shafik AA, el-Sibai O, Ahmed I. Colosigmoid junction: a study of its functional activity with identification of a physiologic sphincter and involvement in reflex actions. J Invest Surg 2003; 16 : 29-34.

5. Shafik A, Asaad S, Doss S. Identification of a sphincter at the sigmoidorectal canal in humans: histomorphologic and morphometric studies. Clin Anat 2003; 16: 138-143.

6. Shafik A. The hypertonic rectosigmoid junction: description of a new clinicopathologic entity causing constipation. Surg Laparosc Endosc 1997; 7: 116-120.

7. Shafik A, Shafik AA, El Sibai O, Ahmed I, Mostafa RM. Role of the rectosigmoidal junction in fecal continence: concept of the primary continent mechanism. Arch Surg 2006; 141: 23-26.

8. Levin MD. Pathophysiology and diagnosis of descending perineum syndrome in children. Pelviperineology 2018; 37: 52-56.
9. Levin MD. Reaction to Koppen et al., 'Assessing colonic anatomy normal values based on air contrast enemas in children younger than 6 years'. Pediatr Radiol. 2018; 48: 1674-1677.

10. Palamchuk V. Colon sphincters.2017. https://vpalamarchuk.ru/ function/sfinktery-tolstoj-kishki.html

11. Levin MD. Pathophysiology of functional constipation in elderly. Pelviperineology 2019; 38: 53-57.

12. Duhamel B. Physio-pathology of the internal anal sphincter. Arch Dis Child 1969; 44: 377-381.

13. Taylor BM, Beart RW Jr, Phillips SF. Longitudinal and radial variations of pressure in the human anal sphincter. Gastroenterology 1984; 86: 693-697.

14. Penninckx F, Lestar B, Kerremans R. The internal anal sphincter: mechanisms of control and its role in maintaining anal continence. Baillieres Clin Gastroenterol 1992; 6: 193-214.

15. Tsukada Y, Ito M, Watanabe K, et al. Topographic Anatomy of the Anal Sphincter Complex and Levator Ani Muscle as It Relates to Intersphincteric Resection for Very Low Rectal Disease. Dis Colon Rectum 2016; 59: 426-433.

16. Shafik A. A new concept of the anatomy of the anal sphincter mechanism and the physiology of defecation: mass contraction of the pelvic floor muscles. Int Urogynecol J Pelvic Floor Dysfunct 1998; 9: 28-32.

17. Bharucha AE, Fletcher JG, Melton LJ 3rd, Zinsmeister AR. Obstetric trauma, pelvic floor injury and fecal incontinence: a populationbased case-control study. Am J Gastroenterol 2012; 107: 902-911.

18. Jorge JMN, Habr-Gama A. Anatomy and Embryology of the Colon, Rectum, and Anus. In: Wolff B.G. et al. (eds) The ASCRS Textbook of Colon and Rectal Surgery. Springer, New York, NY 2007; 1-22.

19. Raizada V, Bhargava V, Karsten A, Mittal RK. Functional morphology of anal sphincter complex unveiled by high definition anal manometery and three dimensional ultrasound imaging. Neurogastroenterol Motil 2011; 23: 1013-1019.

20. Levin MD, Troyan VV. Anatomy, and physiology of anorectal zone. Hypothesis of continence and defecation. Novosti chirurgii 2009; 17: $105-118$

21. Levin MD. The role of the external anal sphincter in the physiology of the pelvic floor. Pelviperineology 2017; 36:108-112.

22. Shafik A, El-Sibai 0. Effect of pelvic floor muscle contraction on vesical and rectal function with identification of puborectalisrectovesical inhibitory reflex and levator-rectovesical excitatory reflex. World J Urol 2001; 19: 278-284.

23. Li D, Guo M. Morphology of the levator ani muscle. Dis Colon Rectum 2007; 50: 1831-1839.

24. Bush M, Petros P, Swash M, Fernandez M, Gunnemann A. Defecation 2: Internal anorectal resistance is a critical factor in defecatory disorders. Tech Coloproctol 2012; 16: 445-450.

25. Palit S, Lunniss PJ, Scott SM. The physiology of human defecation. Dig Dis Sci 2012; 57: 1445-1464. 
26. Musial F, Crowell MD. Rectal adaptation to distention: implications for the determination of perception thresholds. Physiol Behav 1995; 58: 1145-1148.

27. Spencer NJ, Dinning PG, Brookes SJ, Costa M. Insights into the mechanisms underlying colonic motor patterns. J Physiol 2016; 594: 4099-4116.

28. Cormack DH. Ham's Histology. By edition A.W. Ham and D.H. Cormack Eighth Edition. 1979. JB. Lippincoft Company.

29. Parks AG, Porter NH, Hardcastle J. The syndrome of the descending perineum. Proc R Soc Med 1966; 59: 477-482.

30. PORTER NH. A physiological study of the pelvic floor in rectal prolapse. Ann R Coll Surg Engl 1962; 31: 379-404.
31. Cheeney G, Nguyen M, Valestin J, Rao SS. Topographic and manometric characterization of the recto-anal inhibitory reflex. Neurogastroenterol Motil. 2012; 24: 147-154.

32. Coloproctology and the pelvic floor.2nd ed. By M.M. Henry and M. Swash. Butterworths. London. 1985.

33. Shafik A. The hypertonic rectosigmoid junction: description of a new clinicopathologic entity causing constipation. Surg Laparosc Endosc 1997; 7: 116-120.

34. Levin MD. Functional constipation in children: Is there a place for surgical treatment. J Pediatr Surg 2019; 54: 616-617.

35. Levin MD. K rentgenologicheskor̆ anatomii tolstoĭ i priamoĭ kishok $u$ deter [Roentgenologic anatomy of the colon and rectum in children]. Vestn Rentgenol Radiol 1985; 2: 40-45. 